\title{
INMACULADA AGUILAR CIVERA Y LA HISTORIA DE LA INGENIERÍA CIVIL
}

\author{
Luis Arciniega García \\ Universitat de València
}

En primer lugar, permítanme manifestar que me siento, por un lado, honrado por formar parte de un acto académico de elogio a una compañera que realmente se lo merece, lo que supone un reconocimiento para la persona, así como un ejemplo para el resto; $y$, por otro, entusiasmado por ahondar en temas historiográficos, hacia los que hay cierto desdén, pues la Historia del Arte en España se muestra algo renuente a estudiar y reflexionar sobre su génesis, devenir, actualidad y perspectivas. En este caso, el acercamiento que hago se realiza desde el encomio, un importante ejercicio de oratoria que debe fluir por los cauces de la síntesis entre la generosidad del análisis y la moderación del mismo para no convertirlo en banal. Ante este reto me siento algo abrumado por la posibilidad de no ser capaz de llevar a buen término el encargo, y me excuso por ocupar el lugar de personas mucho más capacitadas para esta labor.

\section{DATOS BIOGRÁFICOS}

Inmaculada nació en Valencia el 8 de mayo de 1952, hija de los también valencianos José Aguilar (1924-2006) y Concepción Civera (1925-). A los diez años de edad Inmaculada dejó la capital del Turia por las obligaciones profesionales de su padre, prestigioso catedrático de Física Aplicada (Termología), en la Universidad de Murcia primero, y en la Universidad Complutense después. En el hogar, Inmaculada tuvo un ejemplo universitario y científico de alto rango. Entre las condecoraciones y los puestos de José Aguilar simplemente referiré el de académico de Número de la Real Academia Nacional de Medicina, sillón $n^{\circ} 20$ - Ciencias Físicas, que tomó posesión en 2003 con el discurso de ingreso: "El efecto invernadero, el cambio climático, la crisis medioambiental y el futuro de la Tierra". Y selecciono este por producirse en un año especialmente grato en lo profesional para la familia, pues si el padre recibía un importante reconocimiento a su trayectoria profesional con su ingreso en la Academia, la hija firmaba la creación de una cátedra institucional que ha dirigido hasta su jubilación en 2017. 
La señalada vinculación profesional paterna, hizo que Inmaculada Aguilar estudiase en Madrid. En el mismo campus universitario, por el azar de la vecindad, gozó de la tutela del insigne historiador José María Jover Zamora (1920-2006), catedrático de Historia Universal Moderna y de España en la Universidad de Valencia (1949-1964) y en la Universidad Complutense (19641974), y después catedrático de Historia Universal Contemporánea en esta última institución hasta su jubilación en 1986. Además, fue elegido miembro numerario de la Real Academia de la Historia en 1978, con toma de posesión en 1982. José María Jover, de producción amplia y diversa en lo cronológico, temático y metodológico, con clara impronta de la escuela de los Annales y otras corrientes europeas, ejerció una tutela extraoficial sobre los estudios de licenciatura de Historia del Arte de Inmaculada Aguilar. Estos finalizaron en 1976, en unos años convulsos en los que se abrían corrientes de renovación y apertura. Sirva como ejemplo que un año antes, en el que murió el dictador Francisco Franco, José María Jover recibió el encargo de dirigir la Historia de España, fundada e iniciada por Ramón Menéndez Pidal.

Para la nueva etapa de exigencia investigadora, José María Jover puso a Inmaculada Aguilar en contacto con Pedro Navascués Palacio (1942-), auténtica autoridad en España sobre Historia de la Arquitectura, con especial atención a la del siglo XIX. Desde 1964 ejercía como profesor en la Facultad de Filosofía y Letras de la Universidad Complutense de Madrid, así como de la Escuela Técnica Superior de Arquitectura de la Universidad Politécnica de Madrid, en la que en 1978 obtuvo la cátedra de Historia del Arte. Un año antes de este reconocimiento, bajo su dirección Inmaculada Aguilar presentó la tesina "La obra arquitectónica de Demetrio Ribes Marco", que obtuvo la calificación de Sobresaliente. El binomio continuó en la preparación del siguiente reto, la Tesis Doctor. Como bien sabe el lector, una Tesis Doctoral siempre exige una dirección, pero en menos ocasiones cuenta con una maestría. Inmaculada Aguilar, desde entonces y hasta nuestros días ha tenido en Pedro Navascués un maestro y un mentor.

La sólida formación recibida apuntaba hacia una vida profesional en Madrid, pero por giro personal su trayectoria comenzó y se desarrolló en el departamento de Historia del Arte de la Universitat de València. Primero, como Ayudante C.P., desde el curso 1982/1983 hasta finales de 1986. Bajo la dirección en el citado departamento de Santiago Sebastián López, en 1987 Inmaculada Aguilar defendió la Tesis Doctoral "Las estaciones de ferrocarril en España: tipología y evolución. La Compañía de los Caminos de Hierro del Norte", dirigida por Pedro Navascués Palacio, y que obtuvo la máxima calificación; en aquel momento, Apto Cum Laude. 
En el citado año de 1987, en la institución valenciana ocupó puestos como los de Profesora Colaboradora, Titular-Interina y Titular de Universidad. Precisamente en este tiempo conocí a Inmaculada Aguilar como docente, en aquél momento al frente de la asignatura anual de "Arte Griego" en los años de especialización en Historia del Arte en la licenciatura de Geografía e Historia. Con el tiempo pudo elegir asignaturas más afines a su línea de investigación, como "Arqueología Industrial" o "Últimas Tendencias", de las que ha dejado grato recuerdo y hasta huella en varias generaciones. Con el tiempo, su presencia en asignaturas de contemporáneo se afianzó, y a comienzos de la década de los noventa tuve la oportunidad de disfrutar en el doctorado sus clases sobre arquitectura industrial.

La trayectoria investigadora de la profesora Aguilar y la transferencia de la misma a la sociedad han tenido amplio reconocimiento. Así, en 2002 fue nombrada académica correspondiente de la Real Academia de Bellas Artes de San Fernando, y a finales del año 2003 firmó con instituciones de la Generalitat Valenciana la cátedra institucional de la Universitat de València "Demetrio Ribes", que ha dirigido hasta 2017 y en la que permanece como directora honorífica. En este lapso, en el año 2011 por concurso oposición pasó a ocupar el puesto de Catedrática de Universidad.

En septiembre de 2017 Inmaculada Aguilar se jubiló, no por falta de ilusión o capacidad, sino ante una coyuntura adversa de salud provocada por numerosas horas de sedentarismo entre pantallas de ordenador, libros y expedientes. En este sentido, resulta elocuente que algunos de los recientes manuales dirigidos a la enseñanza universitaria acaben con un capítulo de consejos al docente sobre el control de su ansiedad, la necesidad de cuidar su salud y llevar una vida con hábitos saludables. Unos consejos que Inmaculada Aguilar sigue ahora de manera disciplinada, y que están teniendo tales efectos que parece que asistiéramos a la versión valenciana y en femenino del relato de F. Scott Fitzgerald, The curious case of Benjamin Button (1921). De este nuevo periodo los mayores beneficiados, además de ella misma, son sus seres más queridos y dispersos geográficamente, su madre, su marido, su hija Inmaculada y sus nietos.

\section{LÍNEAS DE INVESTIGACIÓN}

En el caso de Inmaculada Aguilar, la palabra "línea" tiene plena coherencia en la acepción que habla de "dirección, tendencia, orientación de un arte o de un saber". Frente a la dispersión que cultivamos muchos investigadores, la orientación de su investigación se ha mantenido desde los inicios. En su caso, 
sería apropiado no solo hablar de línea de investigación, sino de línea férrea, por cuanto esta expresión evoca la firmeza de su trabajo y alude a su primera línea de investigación (la ferroviaria), que se ha ampliado con otras, claramente complementarias e interrelacionadas. A continuación, esbozaré las tres grandes líneas de su investigación con algunas de sus principales aportaciones.

\section{Historia de la arquitectura ferroviaria en España}

A partir de los estudios iniciados en su Tesina y su Tesis Doctoral, su contribución en este sentido ha sido abrumadora, con numerosas obras que abordan al arquitecto e ingeniero Demetrio Ribes, como Demetri Ribes (1980); Demetrio Ribes, arquitecto 1875-1921 (2004); La mirada del arquitecte. Demetrio Ribes i la seua càmara estereoscòpica (2007)... Y otras que se acercan a las estaciones de ferrocarril, como Historia de las estaciones. Arquitectura ferroviaria en Valencia (1984); La estación de ferrocarril, puerta de la ciudad (1988); Estaciones y ferrocarriles valencianos (1995); Caminos de hierro, estaciones, puentes, viaductos y locomotoras (2005); Encuentros en la estación. València-Nord, un espacio de modernidad (1917-2017) (2017).

\section{Arquitectura Industrial}

En este ámbito de estudio, Inmaculada Aguilar ha sido realmente pionera y activista desde la última década del siglo XX, haciéndose eco de manera muy temprana de las recomendaciones emanadas en 1990 por la Comisión Europea sobre Protection and conservation of the industrial, technical and civil engineering heritage in Europe. Así lo muestran publicaciones como El orden industrial en la ciudad. Valencia en la segunda mitad del siglo XIX (1990); Arquitectura industrial. Concepto, método y fuentes (1998)..., y su labor promotora y asociacionista. En este sentido, debe destacarse que fue socia-fundadora y presidenta de la Asociación Valenciana de Arqueología industrial (1994-1999) y ha sido vocal de la Asociación para la defensa del patrimonio industrial, TICCIH ESPAÑA (2003-2009). Finalmente, con especial atención por su trascendencia patrimonial, cabe destacar que formó parte de la Comisión delegada del Consejo de Patrimonio Histórico Español para la elaboración del Plan Nacional de Patrimonio Industrial (2001-2003). 


\section{Ingeniería civil}

Esta línea, que en gran medida comprende las dos anteriores, se ha desarrollado de manera más amplia desde la cátedra institucional de la Universitat de València "Demetrio Ribes", que Inmaculada Aguilar fundó en el año 2003. Desde la misma ha realizado una constante labor de investigación y transferencia, así como de colaboración con numerosas instituciones afines; por ejemplo, desde 2005 a 2010 fue miembro del consejo asesor del Centro de Estudios Históricos de Obras Públicas y Urbanismo, Ministerio de Fomento. En la actualidad es miembro de la comisión asesora de la Fundación Juanelo Turriano, colaboradora de la Fundación Miguel Aguiló y directora honorífica de la Catedra Demetrio Ribes.

La creación de la citada cátedra institucional estuvo acompañada con la publicación del libro El territorio como proyecto. Transporte, obras públicas y ordenación territorial en la historia de la Comunidad Valenciana (2003). Este puede entenderse como una declaración de la filosofía de trabajo de la cátedra; esto es, la historia de las infraestructuras, viarias -caminos, carreteras, ferrocarriles- portuarias, hidráulicas, etc., y sus interrelaciones; la evolución del estudio de la ingeniería en España y de sus principales artífices, la transferencia de tecnología a lo largo de las diferentes épocas históricas, etc. El paisaje transformado es el reflejo de intereses y necesidades muy diversas a lo largo de la historia de un territorio y de la sociedad que lo ha ido transformando, que ha ido creando unas redes de comunicación muy variadas, que se interrelacionan entre sí para dar respuestas a las necesidades de una sociedad en perpetua transformación. Muchas obras se han sucedido desde entonces, escritas, dirigidas o coordinadas por Inmaculada Aguilar; por ejemplo, 100 Elementos del paisaje valenciano. Las Obras Públicas (2005); Construir, registrar y representar. Sendas, caminos y carreteras de la Comunitat Valenciana. Mapas y planos 1550-1850 (2009); La fachada litoral. Naturaleza y artificio. Mapas, planos y vistas de la Comunidad Valenciana, 1550-1868 (2012); El discurso del ingeniero en el siglo XIX. Aportaciones a la historia de las obras públicas (2012).

\section{LABOR DE GESTIÓN Y TRANSFERENCIA}

Además de una labor docente e investigadora, Inmaculada Aguilar ha tenido una destacada labor de gestión universitaria y de transferencia. En concreto, por un lado, ha sido directora del departamento de Historia del Arte de la Universitat de València desde el 1 de abril de 1995 al 31 de marzo de 1998. 
Un periodo en el que cabe destacar que en 1996 iniciara las "Prácticas Externas". Por otro, ha sido directora de la Cátedra Demetrio Ribes de la Universitat de València, desde diciembre de 2003 hasta su jubilación en 2017, y de la que sigue como directora honoraria. En ella adquiere sentido su investigación, pues la Cátedra tiene por objetivo la investigación, la difusión y el fomento de los estudios sobre la historia del transporte y sus infraestructuras, la obra pública y la ingeniería en la Comunitat Valenciana. Incide en el paisaje antrópico, el paisaje modificado por el ser humano a través de la obra pública que, en su constante transformación por las necesidades de la sociedad, se convierte en elemento sensible de desaparición. Desde la Cátedra Demetrio Ribes, por un lado, ha realizado una intensa labor de transferencia del conocimiento como herramienta del desarrollo social e instrumento básico para el sector productivo, en gran medida mediante el comisariado de numerosas exposiciones. Por otro, ha emprendido una ingente labor de estudio de áreas en los límites de la disciplina, puesto que han sido incorporadas muy recientemente como materias prioritarias de estudio dentro del patrimonio.

\section{GAUDEAMUS IGITUR (ALEGRÉMONOS)}

La profesora Inmaculada Aguilar es una persona de sólida formación universitaria. En este ámbito ha contado con el ejemplo de tres grandes catedráticos de Universidad. Don José Aguilar, el eminente físico y dilecto padre con el que las reuniones familiares culminaban entonando el Gaudeamus igitur; don José María Jover, el ilustre historiador, casual vecino de campus universitario y tutor en la sombra durante la licenciatura; y don Pedro Navascués, prestigioso historiador de la arquitectura, director, maestro y mentor. A partir de estos pilares, doña Inmaculada Aguilar, catedrática de Historia del Arte, viene desarrollando una intensa y cualificada labor investigadora desde la que ha contribuido a una normalización en la propia comunidad científica y por extensión en la sociedad. Su línea de investigación se ha desarrollado en un mundo mayoritariamente de arquitectos y, sobre todo, de ingenieros, donde la aproximación más frecuente ha sido la tecnológica. Ella ha conseguido introducir al mismo objeto de estudio una perspectiva histórica más socio cultural y ha sido una de las pocas mujeres que ha incidido en estos temas. Esta apreciación puede constatarse fácilmente en cualquier bibliografía y se hace visible en las fotografías de la mayoría de los seminarios y coloquios en los que ha compartido mesa.

Estas páginas las iniciaba reconociendo la satisfacción por poder hablar, con autoimpuesta contención, de una investigadora como Inmaculada Aguilar, 
y me gustaría acabarlas señalando que esta dicha se acrecienta al ver que se trata de una trayectoria abierta, en plena actividad y madurez, y por muchos años. Por ello, gaudeamus igitur.

\section{BIBLIOGRAFÍA SELECCIONADA ${ }^{1}$}

AGUILAR CIVERA, Inmaculada (1980): Demetri Ribes, Valencia, ed. E. Climent, $217 \mathrm{p}$.

AGUILAR CIVERA, Inmaculada (1984): Historia de las estaciones: Arquitectura ferroviaria en Valencia, València, Diputación Provincial de Valencia, 176 p.

AGUILAR CIVERA, Inmaculada (1988): La estación de ferrocarril. Puerta de la ciudad, València, Generalitat Valenciana, 2 vol. 495 p.

AGUILAR CIVERA, Inmaculada (1990): El orden industrial en la ciudad. Valencia en la segunda mitad del siglo XIX, València, Diputación de Valencia, Historia Local /5, $234 \mathrm{p}$.

AGUILAR CIVERA, Inmaculada (1995): Estaciones y Ferrocarriles Valencianos, València, Generalitat Valenciana, Consell Valencià de Cultura, Serie Minor / 23, 144 p.

AGUILAR CIVERA, Inmaculada (1996): L'Almodí de València i els espais del comerç, València, Generalitat Valenciana, Consell Valencià de Cultura, Serie Minor / 32, $118 \mathrm{p}$.

AGUILAR CIVERA, Inmaculada (1998): Arquitectura Industrial. Concepto, método y fuentes, València, Diputación de Valencia, colección Arqueología Industrial-1, $297 \mathrm{p}$.

AGUILAR CIVERA, Inmaculada (1999): El Patrimonio arquitectónico Industrial, Madrid, Cuadernos de Restauración VII, Instituto Juan de Herrera, Escuela Técnica Superior de Arquitectura de Madrid, $40 \mathrm{p}$.

AGUILAR CIVERA, Inmaculada (dir.) (2001): Arte valenciano contemporáneo: una recopilación bibliográfica desde 1976, València, Conselleria de Cultura, Educació i Ciència, $551 \mathrm{p}$.

AGUILAR CIVERA, Inmaculada y VIDAL, J. (coords.) (2002): 150 años de ferrocarril en la Comunidad Valenciana: 1852-2002, València, Conselleria d'Obres Públiques, Urbanisme i Transports, 390 p.

AGUILAR CIVERA, Inmaculada y GARCÍA, V. (2003): Espai ferroviari a Marxalenes: el Trenet a València, València, Ajuntament de València, Delegación de Urbanismo-Parques y Jardines, $76 \mathrm{p}$.

AGUILAR CIVERA, Inmaculada (coord.) (2003): Historia del ferrocarril en las comarcas valencianas: La Ribera Alta, València, Conselleria d'Obres Públiques, Urbanisme i Transports, $185 \mathrm{p}$.

\footnotetext{
${ }^{1}$ Por razones de espacio queda reducida a libros publicados, dirigidos o coordinados.
} 
AGUILAR CIVERA, Inmaculada (2003): El territorio como proyecto. Transporte, obras públicas y ordenación territorial en la historia de la Comunidad Valenciana, València, Conselleria de Obras Públicas, Urbanismo y Transportes, Generalitat Valenciana, $277 \mathrm{p}$.

AGUILAR CIVERA, Inmaculada (coord.), (2004): Historia del ferrocarril en las comarcas valencianas: La Costera, València, Conselleria d'Infraestructures i Transport, $200 \mathrm{p}$.

AGUILAR CIVERA, Inmaculada (2004): Valencia Tranvía 1874-2004, València, Ferrocarrils de la Generalitat Valenciana, 183 p.

AGUILAR CIVERA, Inmaculada (2004): Demetrio Ribes, arquitecto (1875-1921), València, Conselleria d'Infraestructures i Transports, $240 \mathrm{p}$.

AGUILAR CIVERA, Inmaculada (2005): Caminos de hierro, estaciones, puentes, viaductos y locomotoras, València, Colegio de Ingenieros de Caminos, Canales y Puertos, Comunidad Valenciana, $167 \mathrm{p}$.

AGUILAR CIVERA, Inmaculada y GARCÍA, V. (2005): El faro del puerto de Valencia. 75 aniversario (1930-2005), València, Cátedra Demetrio Ribes, UVEG-FGV, Colección Cuadernos del Museo del Transporte de la Comunidad Valenciana. Historia del transporte, obra pública y telecomunicaciones, $\mathrm{n}^{\circ} 1,16 \mathrm{p}$.

AGUILAR CIVERA, Inmaculada y GARCÍA, V. (2005): Las estaciones de Carlet y Campamento de Paterna. Puesta en valor del patrimonio de Ferrocarrils de la Generalitat Valenciana, València, Cátedra Demetrio Ribes, UVEG-FGV, Colección Cuadernos del Museo del Transporte de la Comunidad Valenciana. Historia del transporte, obra pública y telecomunicaciones, $\mathrm{n}^{\circ} 3,23 \mathrm{p}$.

AGUILAR CIVERA, Inmaculada (dir.) (2005): Cien elementos del paisaje valenciano: las obras públicas, València, Generalitat Valenciana, Conselleria d'Infraestructures i Transport, $304 \mathrm{p}$.

AGUILAR CIVERA, Inmaculada (coord.) (2006): Benicàssim y la historia del ferrocarril, València, Conselleria d'Infraestructures i Transport, $219 \mathrm{p}$.

AGUILAR CIVERA, Inmaculada (2006): De la Refinería La Británica a la Factoría "La Cantera" de Alicante, València, Cátedra Demetrio Ribes UVEG-FGV, Colección Cuadernos del Museo del Transporte de la Comunidad Valenciana. Historia del transporte, obra pública y telecomunicaciones, $\mathrm{n}^{\circ} 2,40 \mathrm{p}$.

AGUILAR CIVERA, Inmaculada (coord.) (2007): El tranvía de Alicante: pasado y futuro, Valencia, Conselleria de Infraestructuras y Transporte, 238 p.

AGUILAR CIVERA, Inmaculada (coord.) (2007): Historia del ferrocarril en las comarcas valencianas: La Plana Utiel-Requena, València, Conselleria d'Infraestructures i Transport, $249 \mathrm{p}$.

AGUILAR CIVERA, Inmaculada y FERNÁNDEZ, J. A. (2007): La mirada de l'arquitecte. Demetrio Ribes i la seua càmera estereoscòpica, València, Universitat de València, 297 p.

AGUILAR CIVERA, Inmaculada (2007): Arquitecturas del TRAM, talleres y cocheras de El Campello, València, Cátedra Demetrio Ribes UVEG-FGV, Colección Cuadernos del Museo del Transporte de la Comunidad Valenciana. Historia del transporte, obra pública y telecomunicaciones, $\mathrm{n}^{\circ} 5,40 \mathrm{p}$. 
AGUILAR CIVERA, Inmaculada y FERRER, J. (2008): El faro de Alicante. Una recuperación del patrimonio portuario. València, Cátedra Demetrio Ribes, UVEGFGV, Colección Cuadernos del Museo del Transporte de la Comunidad Valenciana. Historia del transporte, obra pública y telecomunicaciones, $\mathrm{n}^{\circ} 6,32 \mathrm{p}$.

AGUILAR CIVERA, Inmaculada (2008): La estación del Trenet. Puerta de la Marina. Historia y puesta en valor del patrimonio de $F G V$, València, Cátedra Demetrio Ribes, UVEG-FGV, Colección Cuadernos del Museo del Transporte de la Comunidad Valenciana. Historia del transporte, obra pública y telecomunicaciones, $\mathrm{n}^{\circ} 7$, 2008, 48 p.

AGUILAR CIVERA, Inmaculada (2008): El TRAM de Castellón. Un paseo por la ciudad, València, Cátedra Demetrio Ribes, UVEG-FGV, Colección Cuadernos del Museo del Transporte de la Comunidad Valenciana. Historia del transporte, obra pública y telecomunicaciones, $48 \mathrm{p}$.

AGUILAR CIVERA, Inmaculada (2008): Itinerarios históricos: el Camino Real del Reyno de Valencia, València, Cátedra Demetrio Ribes, UVEG-FGV, Colección Cuadernos del Museo del Transporte de la Comunidad Valenciana. Historia del transporte, obra pública y telecomunicaciones, $\mathrm{n}^{\circ} 9,44 \mathrm{p}$.

AGUILAR CIVERA, Inmaculada y OLLER, J. (2008): El primer vuelo a motor en España. Paterna, 1909, València, Cátedra Demetrio Ribes, UVEG-FGV, Colección: Cuadernos del Museo del Transporte de la Comunidad Valenciana. Historia del transporte, obra pública y telecomunicaciones, $\mathrm{n}^{\circ} 10,48 \mathrm{p}$.

AGUILAR CIVERA, Inmaculada (coord.) (2009): 100 años de historia de la aviación en la Comunidad Valenciana, Valencia, València, Conselleria d'Infraestructures $i$ Transport, $469 \mathrm{p}$.

AGUILAR CIVERA, Inmaculada (2009): Construir, registrar y representar. Sendas, caminos y carreteras de la Comunitat Valenciana. Mapas y planos 1550-1850, València, Conselleria d'Infraestructures i Transport, 362 p.

AGUILAR CIVERA, Inmaculada (2009): El ingeniero industrial Vicente Pichó. Una visión renovadora de la ciudad. Colección Cuadernos del Museo del Transporte de la Comunidad Valenciana. Historia del transporte, obra pública y telecomunicaciones, $\mathrm{n}^{\circ}$ 11, València, Cátedra Demetrio Ribes, UVEG-FGV, 48 p.

AGUILAR CIVERA, Inmaculada (2010): The mediterranean corridor: 2000 years uniting europe = Le Corridor Mediterraneen: Unissant l'Europe depuis 2000 ans = El Corredor Mediterráneo. 2000 años de historia uniendo Europa, Catálogo de la exposición. Edificio Jacques Delors, Comité Económico y Social (Bruselas), València, Generalitat Valenciana, $80 \mathrm{p}$.

AGUILAR CIVERA, Inmaculada (2010): Arquitecturas del TRAM. La estación de Luceros: nueva plaza en la ciudad, Colección Cuadernos del Museo del Transporte de la Comunidad Valenciana. Historia del transporte, obra pública y telecomunicaciones, València, Conselleria d'Infraestructures i Transport/Cátedra Demetrio Ribes UVEG-FGV, $\mathrm{n}^{\circ} 12,48 \mathrm{p}$.

AGUILAR CIVERA, Inmaculada (2010): “Aportaciones a la historia del lenguaje técnico. Pelayo Clairac y el Diccionario de Arquitectura e ingeniería”, en Clairac y 
Sáenz, Pelayo, Diccionario General de Arquitectura e Ingeniería (1877-1908), Madrid, Ministerio de Fomento, CEDEX-CEHOPU, pp. 7-45.

AGUILAR CIVERA, Inmaculada (2010): Puente de Astilleros. El legado de la ingeniería de Caminos, Canales y Puertos: obras y documentos. València, Colegio de Ingenieros de Caminos, Canales y Puertos de la Comunidad Valenciana, $160 \mathrm{p}$.

AGUILAR CIVERA, Inmaculada (ed.) (2010): Diccionario General de Arquitectura e Ingeniería de Pelayo Clairac, Madrid, Ministerio de Fomento, CEDEX-CEHOPU, recurso electrónico y folleto de 45 p.

AGUILAR CIVERA, Inmaculada (coord.) (2010): Guía de puertos de la Comunitat Valenciana, València, Generalitat Valenciana, Conselleria d'Infraestructures i Transport, $326 \mathrm{p}$.

AGUILAR CIVERA, Inmaculada (2011): Mar y tierra. Los escenarios del Grao de Valencia (catálogo de exposición), València, Generalitat Valenciana, 88 p.

AGUILAR CIVERA, Inmaculada (coord.) (2011): AVE Valencia - Madrid: diálogos sobre el territorio y escenas desde el tren, Valencia, Conselleria d'Infraestructures, Territori i Medi Ambient, 216 p.

AGUILAR CIVERA, Inmaculada (2012): Historia del ferrocarril en las comarcas valencianas: El Camp de Morvedre, València, Conselleria d'Infraestructures i Transport, $224 \mathrm{p}$.

AGUILAR CIVERA, Inmaculada (2012): El discurso del ingeniero en el siglo XIX. Aportaciones a la Historia de las Obras Públicas, Madrid, Fundación Juanelo Turriano, Conselleria de Infraestructuras, Territorio y Medio Ambiente, Generalitat Valenciana, $312 \mathrm{p}$.

AGUILAR CIVERA, Inmaculada (2012): Lugares e itinerarios de las obras públicas. El paisaje valenciano, (catálogo de exposición), València, Conselleria de Infraestructuras, Territorio y Medio Ambiente, 103 p.

AGUILAR CIVERA, Inmaculada (2012): La fachada litoral. Naturaleza y artificio. Mapas, cartas, planos y vistas de la Comunitat Valenciana, 1550-1868, València, Conselleria de Infraestructuras, Territorio y Medio Ambiente, 346 p.

AGUILAR CIVERA, Inmaculada (2013): Los Docks Comerciales del puerto de Valencia. Una obra centenaria, Colección Cuadernos del Museo del Transporte de la Comunidad Valenciana. Historia del transporte, obra pública y telecomunicaciones, València, Conselleria d'Infraestructures i Transport, Cátedra Demetrio Ribes, UVEG-FGV, no 16, 44 p.

AGUILAR CIVERA, Inmaculada; MUÑOZ, D. y URZAINQUI, S. (2013): Caminos a Castilla. Paisajes del transporte, (catálogo de exposición) València, Conselleria de Infraestructuras, Territorio y Medio Ambiente, 88 p.

AGUILAR CIVERA, Inmaculada y FERRER, J. (coords.) (2013): El comercio y la cultura del mar. Alicante, puerta del Mediterráneo, València, Conselleria d'Infraestructures, Territori i Medi Ambient, $456 \mathrm{p}$.

AGUILAR CIVERA, Inmaculada (coord.) (2014): Luces y faros del Mediterráneo: paisaje, técnica, arte y sociedad de Torrevieja a Vinaròs, València, Conselleria d'Infraestructures, Territori i Medi Ambient, 279 p. 
AGUILAR CIVERA, Inmaculada y BOLINCHES, L. (2015): El Edificio del Reloj. Símbolo del puerto de Valencia, una obra centenaria. València, Conselleria d'Habitatge, Obres Públiques i Vertebració del Territori , Cátedra Demetrio Ribes UVCHOPVT, $44 \mathrm{p}$.

AGUILAR CIVERA, Inmaculada y ROCA, A. (dirs.) (2015): 30 años construyendo puentes: técnica e innovación, València, Conselleria d'Infraestructures, Territori i Medi Ambient, 247 p.

AGUILAR CIVERA, Inmaculada (2015): El ingeniero Lucio del Valle en el distrito de Valencia: El manuscrito inédito de su correspondencia, 1842-1846. Una aportación a la historia de la ingeniería. València, Generalitat Valenciana, Conselleria d'Habitatge, Obres Públiques i Vertebració del Territori, 133 p.

AGUILAR CIVERA, Inmaculada (2015): Los paisajes del agua. Cultura, transporte y territorio. Mapas y planos de la Comunitat Valenciana, 1550-1868, València, Conselleria de Infraestructuras, Territorio y Medio Ambiente, 316 p.

AGUILAR CIVERA, Inmaculada (2016): Mirades i testimonis: València marítima (catálogo de exposición), València, Conselleria d'Habitatge, Obres Públiques i Vertebració del Territori, 87 p.

AGUILAR CIVERA, Inmaculada y AGUILÓ, M. (coords.) (2016): Guía de puentes de la Comunitat Valenciana: 2016, Valencia, Conselleria d'Habitatge, Obres Públiques i Vertebració del Territori, 158 p. y una memoria USB.

AGUILAR CIVERA, Inmaculada (2017): Los varaderos del puerto de València: una historia centenaria, Colección: Cuadernos del Museo del Transporte de la Comunidad Valenciana. Historia del transporte, obra pública y telecomunicaciones, 25. València, Conselleria d'Habitatge, Obres Públiques i Vertebració del Territori, 48 p.

AGUILAR CIVERA, Inmaculada y SERRA, A. (dirs.) (2017): Los poblados marítimos: historia, lugares y escenas, València, Ajuntament de València, Cátedra Demetrio Ribes, $349 \mathrm{p}$.

AGUILAR CIVERA, Inmaculada (2017): Encontres en l'estació: València-Nord, un espai de modernitat (1917-2017) = Encuentros en la estación: València-Nord, un espacio de modernidad (1917-2017), València, Ajuntament de València, Regidoria de Patrimoni Cultural i Recursos Culturals, Càtedra Demetrio Ribes, 213 p. 
